\title{
Small Cell Carcinoma with Paraneoplastic Polyneuropathy and Tumor Embolization: A Case Report and Literature Review
}

\author{
Manica Sodhi ${ }^{\mathrm{b}}$ James K. Stoller ${ }^{\mathrm{a}, \mathrm{c}}$

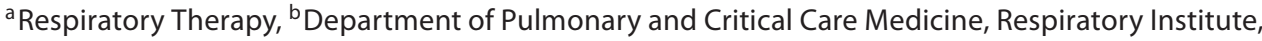 \\ Cleveland Clinic, and ${ }^{\mathrm{C} C l e v e l a n d}$ Clinic Lerner College of Medicine, Cleveland, Ohio, USA
}

\section{Established Facts}

- Paraneoplastic sensory polyneuropathy with anti-Hu antibodies is an uncommon manifestation of cancer which is most frequently associated with small cell carcinoma.

- Available estimates suggest that fewer than $1 \%$ of all neoplasms and $4 \%$ of small cell cancers are complicated by paraneoplastic sensory polyneuropathy.

\section{Novel Insights}

- This patient represents only the second individual to be described with tumor embolization complicating small cell cancer and reminds clinicians of the extended spectrum of this disease.

\section{Key Words}

Small cell cancer $\cdot$ Tumor embolism · Sensory

polyneuropathy $\cdot$ Anti-Hu antibodies

\begin{abstract}
Although small cell cancer of the lung may have protean manifestations, tumor embolization and inability to identify the primary sites are unusual features. We present a patient with anti-Hu antibody-associated paraneoplastic sensory polyneuropathy and tumor embolism diagnosed by endovascular biopsy to be due to small cell cancer, the primary site of which was not evident. To our knowledge, this patient
\end{abstract}

\section{KARGER}

Fax +41613061234 E-Mail karger@karger.ch www.karger.com (c) 2009 S. Karger AG, Basel 0025-7931/10/0791-0077\$26.00/0

Accessible online at: www.karger.com/res represents only the second individual to be described with tumor embolization complicating small cell cancer and reminds clinicians of the extended spectrum of this disease.

Copyright $\odot 2009$ S. Karger AG, Basel

\section{Introduction}

Paraneoplastic sensory polyneuropathy (PSP) with anti-Hu antibodies (AHA) is an uncommon manifestation of cancer $[1,2]$ which is most frequently associated with small cell carcinoma (SCC) [3, 4]. Hematogenous embolization is another uncommon complication of 

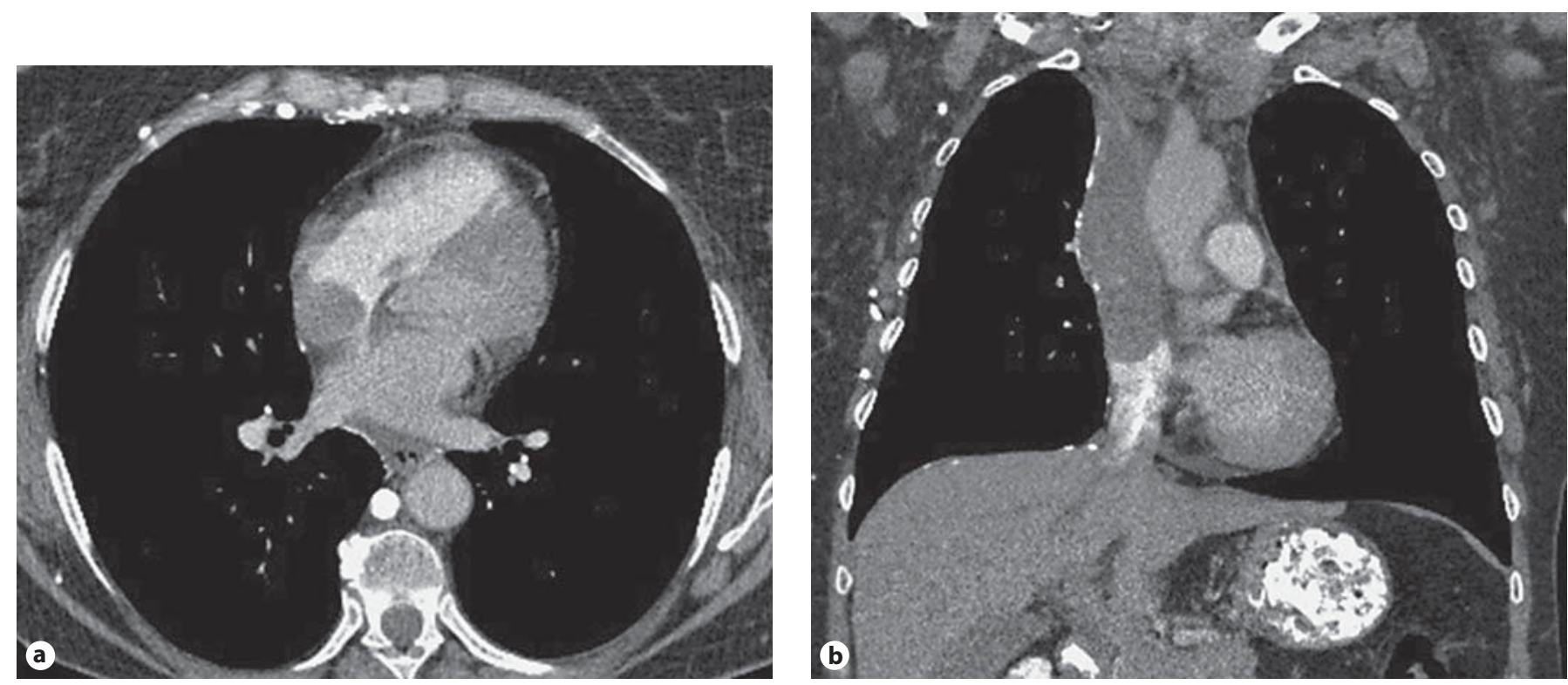

Fig. 1. a Sagittal CT section showing a filling defect at the level of the right atrium. Endovascular biopsy of the filling defect showed SCC. $\mathbf{b}$ Coronal section from the patient's chest CT showing a vascular filling defect extending from the right atrium to the junction of the innominate veins, where the endovascular biopsy was taken.

cancer [5-7] which is rare in the setting of SCC [8]. To demonstrate several unusual features of SCC, this report describes a woman who presented with a polyneuropathy and superior vena cava (SVC) syndrome and was found to have AHA and hematogenous embolization of SCC.

\section{Case Report}

A 52-year-old woman was transferred to the Cleveland Clinic with a 10-month history of hyperesthesia of the extremities and more recent facial swelling.

On physical examination, she had facial fullness. Her chest was clear, cardiac and abdominal examinations were unremarkable, and she had no palpable lymphadenopathy. Extremities showed no cyanosis, clubbing or edema. Neurological examination showed marked hyperesthesia of all extremities from the ankles to the knees and from her fingers to her elbows.

Laboratory evaluation showed normal complete blood count and electrolytes. Outside studies included a chest X-ray showing a right paratracheal mass and calcified parenchymal granulomata. Before transfer, MRI of the brain and spine showed no masses and small disk herniations (T7-8 and L5-S1). Chest CT (fig. 1) showed an intraluminal filling defect (extending from the junction of the innominate veins through the SVC to the level of the right atrium) and extensive chest wall collateral vessels. Also, there was retrocaval, right paratracheal and subcarinal lymph- adenopathy (some calcified), ranging from 15 to $30 \mathrm{~mm}$ in diameter but no parenchymal masses other than calcified granulomata. Abdominal CT showed no findings suggesting neoplasm. On suspicion of polyneuropathy, workup included a paraneoplastic antibody panel which showed elevated AHA titers but was otherwise negative. Bronchoscopy showed a normal endobronchial examination. An endovascular biopsy of the filling defect in the innominate veins (fig. 1) showed SCC (fig. 2). home.

The patient elected early discharge to seek treatment closer to

\section{Discussion}

This patient extends the spectrum of described manifestations of SCC in demonstrating both anti-Hu PSP and hematogenous embolization.

Unusual features of our patient's illness include: (1) the presence of PSP due to onconeural antibodies, which has been described in only $1.7 \%$ of patients evaluated for neuropathy [1], (2) an atypical presentation of SCC given the absence of either pulmonary parenchymal or airway abnormalities, and (3) demonstration of tumor embolization by endovascular biopsy in the context of the SVC syndrome. 
Fig. 2. Endovascular biopsy showing sheets of small cells with scant cytoplasm, no apparent cell borders and absence of nucleoli. Frequent mitoses and nuclear molding structures suggestive of rosette formation are also appreciated. The cells were characterized by marked staining for broad spectrum cytokeratins (AE1/ AE3), strong expression of low molecular weight cytokeratins (CAM 5.2) and synaptophysin with scattered cells positive for chromogranin.

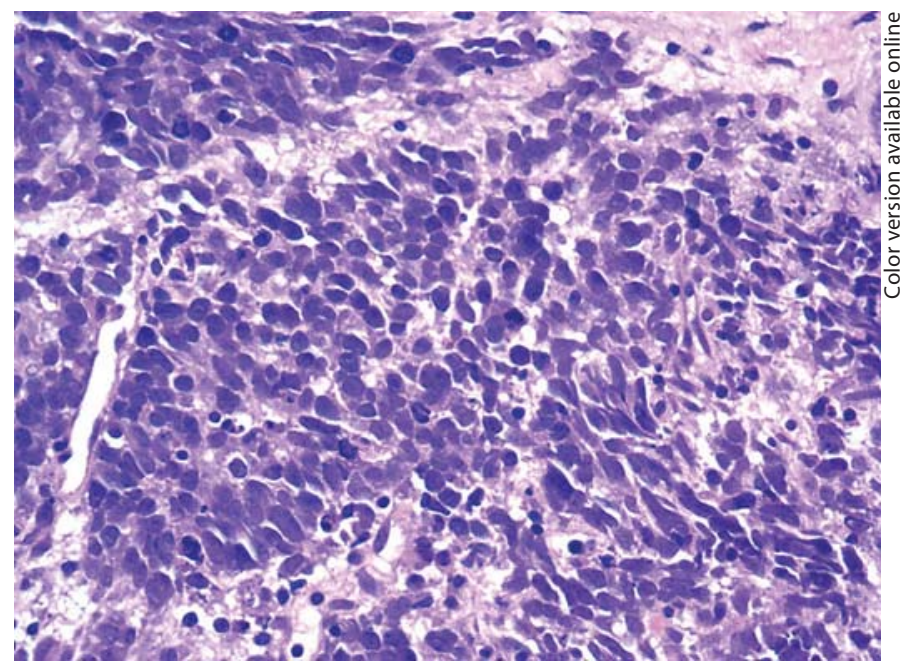

Paraneoplastic neurologic syndromes are caused by autoimmune processes triggered by the cancer and directed against antigens (called 'onconeural antigens') that are common to both the cancer and the nervous system. Such syndromes can affect any part of the central and peripheral nervous system, the neuromuscular junction and muscle and include: Lambert-Eaton myasthenic syndrome, subacute cerebellar ataxia, limbic encephalitis, opsoclonus - myoclonus, retinopathies, sensory neuropathies, encephalomyelitis and dermatomyositis. Among these paraneoplastic neurologic syndromes, the most common is PSP, which accounts for $31-54 \%$ of instances $[1,2]$. PSP is an uncommon complication of cancer, with available estimates suggesting that fewer than $1 \%$ of all neoplasms [3] and 4\% of SCCs [4] are complicated by PSP. Classic clinical manifestations of PSP at onset are pain and paresthesias with asymmetric distribution that involve the arms. Later, pain may be replaced by numbness, limb ataxia and pseudoathetotic movements of the limbs [5].

AHA (i.e. antibodies that are part of a family of RNAbinding proteins - HuD, HuC, Hel-N1, Hel-N2 - which are expressed in the nuclei of neurons and in cancer cells) account for most cases of PSP [1,8]; furthermore, lung SCC accounts for most [3,4] cancers associated with AHA (66-78\%). Other neoplasms (e.g., prostate, adrenal) [1] are less frequently associated with AHA.

Lung SCC typically arises in the central airways, infiltrating the submucosa and gradually obstructing the bronchial lumen through extrinsic or endobronchial spread. A radiographic presentation as a hilar mass with

bulky mediastinal adenopathy is most common. However, notably, individuals with SCC and PSP often experience a long delay between the onset of the neuropathy and initial detection of the tumor (mean $6.5 \pm 7.0$ months, range 1-47) [1,4]. In our patient, though available imaging did not identify a primary SCC site, her truncated hospitalization precluded some testing (e.g. positron emission tomography) that might have revealed a primary site.

Finally, tumor embolization is uncommon in lung cancer, estimated to occur in only $10 \%$ of cases [6-8]. Tumor embolization with lung SCC is even more rare, with only one single case report available [9]; Wong et al. [9] described a 55-year-old man who developed acute dyspnea and hemodynamic collapse found at postmortem to be due to extensive SCC tumor embolism. A second related case [10] regards a patient with SCC that presented as an intravascular tumor in the left main pulmonary artery, but without reported extravascular extension noted at surgery; this second patient also initially presented with paraneoplastic polyneuropathy.

To our knowledge, our patient represents only the second case of SCC complicated by tumor embolization. In this regard, she extends available experience and reminds clinicians that SCC may have protean manifestations, including PSP and vascular occlusion due to tumor embolization. 


\section{References}

$D_{1}$ Graus F, Keime-Guibert F, Rene R, et al: Anti-Hu-associated paraneoplastic encephalomyelitis: analysis of 200 patients. Brain 2001;124:1138-1148.

$\checkmark 2$ Ansari J, Nagabhushan N, Syed R, et al: Small cell lung cancer associated with anti-Hu paraneoplastic sensory neuropathy and peripheral nerve microvasculitis: case report and literature review. Clin Oncol 2004;16: 71-76.

3 Antoine JC, Mosnier JF, Absi L, et al: Carcinoma associated paraneoplastic neurological syndromes. J Neurol Neurosurg Psychiatry 1999;67:7-14.
4 Dalmau J, Graus F, Rosenblum MK, Posner JB: Anti-Hu-associated paraneoplastic encephalomyelitis/sensory polyneuropathy: a clinical study of 71 patients. Medicine 1992; 71:59-72.

5 Kuntzer T, Antoine JC, Steck AJ: Clinical features and pathophysiological basis of sensory neuronopathies (ganglionopathies). Muscle Nerve 2004;30:255-268.

6 Gonzalez-Vitale JC, Garcia-Bunuel R: Pulmonary tumor emboli and cor pulmonale in primary carcinoma of the lung. Cancer 1976; $38: 2105-2110$
7 Morgan AD: The pathology of subacute cor pulmonale in diffuse carcinomatosis of the lungs. J Pathol Bacteriol 1949;61:75-84.

$\checkmark 8$ Onuigbo WI: The carriage of cancer cells by the thoracic duct. Br J Cancer 1967;21:496500 .

$\checkmark$ Wong CH, Suvarna SK, Ciulli F, Leggett RJE: Small cell lung carcinoma presenting as acute cardiovascular collapse due to tumor cell embolisation. Respiration 2000;67:323326.

10 Teo TKB, Venkatesh SK, Tan LKA, Wong PS: Small cell neuroendocrine carcinoma presenting as a pulmonary artery mass. Clin Radiol 2007;62:1015-1018. 